\title{
An improved readout topology of resistive sensor array for reduced measurement error
}

\begin{abstract}
Readout circuit integrated with large scale sensors array is important for improving the effectiveness of data acquisition system. However to design such system, it will require a variety of additional components which contributes to the increment of power consumption. In this paper we proposed an improved method of readout topology for resistive sensor array to reduce the measurement error. A new equation is also proposed to obtain the resistance value. A simulation has been done on $8 \times 8$ matrix sensor array represented by 64 fixed resistors and 8 standard reference resistors. The results show that the optimum reference resistance is $510 \mathrm{q}$ for the value range between $100 \mathrm{q}$ and $100 \mathrm{kq}$, and the improved percentage error is kept to be lower than $5 \%$.
\end{abstract}

Keyword: Readout circuit; Readout topology; Resistive sensor; Sensor array 